\title{
RECHTSCHARAKTER DES POLNISCHEN VERBOTS VON GEMEINSCHAFTLICHEN TESTAMENTEN AUS DER SICHT DES INTERNATIONALEN PRIVATRECHTS
}

\begin{abstract}
Zusammenfassung
Das Verbot gemeinschaftlicher Testamente ist ein weltweit verbreitetes Rechtsinstitut. Die positive Regelung des Instituts des gemeinschaftlichen Testaments ist dagegen eine Seltenheit. In diesem Zusammenhang kommt der Frage der Qualifikation eines fremden Verbots gemeinschaftlicher Testamente, d.h. seiner Zuordnung entweder dem hypothetischen Erbstatut oder dem Formstatut eine besondere Bedeutung zu. In dem vorliegenden Beitrag wird die Ansicht präsentiert, dass die Qualifikation nach legis fori erfolgen soll. Der Rechtscharakter eines fremden Verbots gemeinschaftlicher Testamente hängt also vom Rechtscharakter des polnischen Verbots gemeinschaftlicher Testamente ab, der zu einem Bezugspunkt bei der Qualifikation wird. Darum ist es so wichtig, den Rechtscharakters des polnischen Verbots gemeinschaftlicher Testamente zu bestimmen. Darüber hinaus kann diese Beurteilung für Gerichte anderer Länder von großer Wichtigkeit sein, die bei der Qualifikation des polnischen Verbots gemeinschaftlicher Testamente den Standpunkt der polnischen Rechtslehre und Rechtsprechung in Betracht ziehen werden.
\end{abstract}

\section{Keywords}

Erbrecht - Internationales Privatrecht - Verfügung von Todes wegen - Verbot des gemeinschaftlichen Testaments - Rechtscharakter des polnischen Verbots des gemeinschaftlichen Testaments

* Dr. Tomasz Jasiakiewicz, Nikolaus-Kopernikus-Universität in Toruń, Assistent im Lehrstuhl für Zivil- und Familienrecht. E-Mail: tomjot@umk.pl. 


\section{EINFÜHRUNG}

Laut Art. 942 des Polnischen Zivilgesetzbuches darf das Testament Verfügungen von nur einem Erblasser enthalten. Es wird allgemein angenommen, dass in der genannten Vorschrift das Verbot von gemeinschaftlichen Testamenten geregelt worden ist. Dieses Rechtsinstitut, das sich auf Art. 968 des Französischen Zivilgesetzbuches anlehnt, ist nicht nur für das polnische Recht, sondern auch für Gesetzgebungen zahlreicher anderer Länder charakteristisch. Es ist zugleich ein Konstrukt, das schon immer gewisse Kontroversen ausgelöst hat. Diese beziehen sich insbesondere auf die Gestalt der aktuell geltenden Regelung, die mit der empfindlichen Sanktion der Ungültigkeit im gleichen Maße gemeinschaftliche Verfügungen der einander fremden Personen, wie auch verbundene Verfügungen von Eheleuten belegt. Die genannten Kontroversen haben ihren Ursprung auch im Inhalt des Art. 942 ZGB selbst, der einen gewissen Interpretationsraum lässt. Daher sind auch sehr unterschiedliche Meinungen zum Umfang des polnischen Verbots von gemeinschaftlichen Testamenten anzutreffen. Im Kontext des Internationalen Privatrechts ist es notwendig, den Charakter des polnischen Verbots von gemeinschaftlichen Testamenten $\mathrm{zu}$ beurteilen. Hierzu werden zwei Standpunkte vertreten. Nach dem ersten Konzept gehört das Verbot gemeinschaftlicher Testamente zum Formstatut. Die Anhänger von dem zweiten Konzept sind dagegen der Meinung, dass das Verbot zum hypothetischen Erbstatut zählt.

Das Ziel des vorliegenden Beitrags ist es, den Rechtscharakter des polnischen Verbots von gemeinschaftlichen Testamenten aus der Sicht des Internationalen Privatrechts näherzubringen. Wegen der engen Auffassung von dem Thema werden manche Fragen nur angedeutet, u.a. die Gründe für die Regelung des polnischen Verbots von gemeinschaftlichen Testamenten, und manche völlig übergangen. Hierbei handelt es sich insbesondere um die einzelnen Minderheitskonzepte zum Verbot gemeinschaftlicher Testamente. Es unterliegt aber keinem Zweifel, dass dem erwähnten internationalprivatrechtlichen Aspekt die Analyse der dominierenden Ansicht zum Umfang des polnischen Verbots gemeinschaftlicher Testamente vorangehen soll. 


\section{WESEN DES VERBOtS VON GEMEINSCHAFtLICHEN TESTAMENTEN IM POLNISCHEN RECHT}

Nach der dominierenden und völlig $\mathrm{zu}$ billigenden Meinung ist es gegen die Vorschrift des Art. 942 ZGB1, die Verfügungen von zwei oder mehreren Erblassern in einer Urkunde zu enthalten ${ }^{2}$. Der Umfang des sich auf das Kriterium der einheitlichen Urkunde stützenden Verbots von gemeinschaftlichen Testamenten sieht folgendermaßen aus. Das holographische Testament darf nicht so errichtet werden, dass die Erblasser zuerst ihre Verfügungen niederschreiben und anschließend darunter ihre Unterschriften leisten. Die Situation ist mehr komplex, wenn einer der Erblasser seine Teilnahme an der Testamentserrichtung darauf beschränkt, seine Unterschrift unter die von dem Mittestator niedergeschriebenen Verfügungen zu leisten. Dann wird das Testament des Erblassers, der den Inhalt der Verfügungen niedergeschrieben hat, für ungültig erklärt, nur weil das Verbot des gemeinschaftlichen Testierens verletzt worden ist. Dagegen die Verfügungen des zweiten Testators, der lediglich

\footnotetext{
1 Es ist zu beachten, dass z.B. das schweizerische Recht das Verbot nicht der Regelung des Art. 942 ZPO ähnlich regelt. Es wird jedoch angenommen, dass das Enthalten von Verfügungen in einer Urkunde unzulässig ist, da das gemeinschaftliche Testament gegen die Formvorschriften verstößt. Diese sehen nämlich nicht vor, dass im Rahmen von einer Testamentsurkunde Verfügungen von mehr als einem Erblasser enthalten sind siehe dazu P. Piotet, [in:] M. Gutzwiller, H. Hinderling, A. Meier-Hayoz, H. Merz, P. Piotet, R. Secrétan, W. von Steiger, F. Vischer (Red.), Schweizerisches Privatrecht. Vierter Band. Erbrecht. Erster Halbband, Basel-Stuttgart 1978, S. 190 ff.; S. Wolf, I. Steiner, [in:] R. Süß, U. Haas (Red.), Erbrecht in Europa, Angelbachtal 2004, S. 906.

2 So K. Przybyłowski, Niedopuszczalność wspólnych testamentów [Unzulässigkeit gemeinschaftlicher Testamente], Studia Cywilistyczne [Zivilrechtliche Studien] 1963, B. IV, S. 11-12. Alle von K. Przybyłowski in dem genannten Beitrag präsentierten Ansichten bezogen sich auf die nicht mehr geltende Rechtsverordnung Erbrecht von 1946; siehe Beschluss der sieben Richter des Obersten Gerichts vom 22.03.1971, (Aktenzeichen) III CZP 91/70, Orzecznictwo Sądów Polskich i Komisji Arbitrażowych [Rechtsprechung Polnischer Gerichte und Schiedsausschüsse], Nr. 2, Pos. 26, S. 56; A. Mączyński, Glosa do uchwały siedmiu sędziów Sądu Najwyższego - Izba Cywilna z dnia 22 marca 1971 [Kommentar zum Beschluss der sieben Richter des Obersten Gerichts - Zivilkammer vom 22. März 1971], III CZP 91/70, Orzecznictwo Sądów Polskich i Komisji Arbitrażowych [Rechtsprechung Polnischer Gerichte und Schiedsausschüsse] 1972, Nr. 2, Pos. 26, S. 59; so auch jetzt, wie es scheint, S. Wójcik, F. Zoll, [in:] B. Kordasiewicz (Red.), System prawa prywatnego. Prawo spadkowe. Tom 10 [System des Privatrechts. Erbrecht. Band 10], Warszawa 2013, S. 321; solch ein Umfang des Verbots gilt auch im österreichischen Recht, siehe P. Apathy, [in:] H. Koziol, P. Bydlinski, R. Bollenberger (Red.), Kurzkommentar zum ABGB, Wien 2007, S. 540.
} 
seine Unterschrift geleistet hat, stellen nicht nur eine Verletzung der Verbotsnorm, sondern auch eine Verletzung der Formvorschrift des holografischen Testaments dar, deren wesentliche Eigenschaft die Eigenhändigkeit ist ${ }^{3}$.

Zweifel in der Rechtslehre wecket eine ähnliche Situation, wenn das Testament gemeinschaftlich als Ganzes niedergeschrieben und von einem Erblasser unterzeichnet wird, der andere Erblasser dagegen seine Unterschrift letztendlich nicht leistet. In einer Sache, die vor dem Obersten Gericht verhandelt wurde, war ein Testament mit folgendem Inhalt errichtet worden: „Wir, die Unterzeichnenden Józef und Stanisława B. (...) erklären, dass wir unsere Landwirtschaft als uneingeschränktes Eigentum Aleksander B., unserem Sohn, wohnhaft in (...) übergeben und ihn zum Alleinerben einsetzen“. Das Testament war von Józef B. vollständig niedergeschrieben, unterschrieben und datiert worden. Das Oberste Gericht vertrat den Standpunkt ${ }^{4}$, dass ohne Rücksicht auf den Inhalt eines solchen Testaments, der eindeutig auf die Gemeinschaftlichkeit der Verfügungen hinweisen kann, das Testament gültig ist, da er Verfügungen von nur einem Erblasser enthält. In der Rechtslehre wurde dagegen die Meinung geäußert, dass der dargestellte Standpunkt des Obersten Gerichts ein Zeichen der Einschränkung des Umfangs des Verbots gemeinschaftlicher Testamente ist ${ }^{5}$. In dem genannten Rechtsstreit soll man sich an die Seite der Rechtsprechung stellen. Das Verbot von gemeinschaftlichen Testamenten umfasst nämlich nicht den Fall, in einer Urkunde ein gültiges Testament mit einem Testamentsentwurf $\mathrm{zu}$ verbinden. Zum Verstoß gegen das Verbot kommt es dagegen in der Situation, wenn das gültige Testament mit einem ungültigen Testament wie oben dargestellt verbunden wird. Der Grund für die Ungültigkeit ist dabei unbedeutend. Das kann zum Beispiel der Verstoß gegen den Grundsatz der Persönlichkeit sein, wenn der Erblasser das Testament in der ersten Person Plural errichtet, und dabei sowohl für sich als auch für den Miterblasser als sein Bevollmächtigter unterzeichnet.

\footnotetext{
3 So treffend Przybyłowski, Fußnote 2, S. 19.

$4 \quad$ Siehe Entscheidung des Obersten Gerichts vom 13.01.1956, IV CR 20/56, Orzecznictwo Sądu Najwyższego Izba Cywilna i Izba Karna [Rechtsprechung des Obersten Gerichts Zivilkammer und Strafkammer] 1957, Nr. 3, Pos. 75.

5 Przybyłowski, Fußnote 2, S. 20.
} 
An dieser Stelle soll bemerkt werden, dass die Tatsache selbst, dass die das Testament errichtenden Erblasser ein Blatt Papier verwenden, keinen ausreichenden Grund darstellt, die auf diesem Blatt niedergeschriebenen Verfügungen als gemeinschaftlich anzusehen. Somit können die Erblasser ihre Testamente eines unter dem anderen, eines neben dem anderen oder auf den zwei Seiten desselben Blattes errichten, unabhängig davon, ob die physische Trennung des Blattes durch das Zerschneiden möglich ist ${ }^{6}$.

Im Fall von einem allographischen, einem notariellen sowie einem auf einem polnischen Schiff oder im Flugzeug errichteten Testament wird wiederum als ein Verstoß gegen das Verbot von gemeinschaftlichen Testamenten angesehen, wenn die Erklärungen der Erblasser entsprechend in einem Protokoll, einer notariellen Urkunde oder einem Schreiben enthalten sind 7 .

Damit kommt es bei der allographischen Form nicht zum Verstoß gegen Art. 942 ZGB durch die bloße Tatsache, dass die Erklärungen an demselben Ort, zu derselben Zeit und in Gegenwart von denselben Zeugen abgegeben werden. Ohne Bedeutung sind auch die inhaltliche Verbindung der Verfügungen und der Zeitpunkt der Anfertigung von Protokollen, die sowohl nach Abschluss der Äußerung eines jeden Erblassers, als auch ganz zuletzt, nach der Abgabe von allen Erklärungen entstehen können. Wichtig ist nur, dass jedes Testament in einem getrennten Protokoll enthalten ist. Diese Anforderung ist eingehalten, auch wenn das Protokoll eines jeden Erblassers auf dem Blatt angefertigt wurde, auf dem auch das Protokoll des anderen Testators steht, wobei aber jedes getrennt unterzeichnet und datiert werden muss. Es ist dabei ohne Bedeutung, dass ihre physische Trennung unmöglich ist ${ }^{8}$. Das gilt auch für notarielle und auf dem polnischen Schiff oder im Flugzeug errichtete Testamente. Das letztere stellt im Grunde genommen eine besondere Form des allographischen Testaments dar9.

So treffend Ibidem, S. 20.

So treffend Ibidem, S. 21.

So treffend Mączyński, Fußnote 2, S. 59.

Nach der Ansicht von J.St. Piątkowski, Prawo spadkowe. Zarys wykładu [Erbrecht. Vortragsskizze], Warszawa 1979, S. 133 stellt das während der Reise auf einem polnischen Schiff oder im Flugzeug errichtete Testament eine besondere Form von einem allographischen Testament dar. E. Skowrońska-Bocian, Komentarz do Kodeksu cywilnego. Księga czwarta. Spadki [Kommentar zum Zivilgesetzbuch. Vierter Band. Erbrecht], Warszawa 2011, 
Das Verbot von gemeinschaftlichen Testamenten gilt aber nicht für mündliche Testamente ${ }^{10}$ und für Schreiben, die den Inhalt des mündlichen Testaments festlegen ${ }^{11}$.

Dass von dem polnischen Gesetzgeber in Art. 942 ZGB ausschließlich das Kriterium der einheitlichen Urkunde angenommen wird, hat zur Folge, dass andere Umstände, die auf die Gemeinschaftlichkeit der Verfügungen hinweisen können, im Kontext dieser Vorschrift irrelevant sind. Erblasser, die Verfügungen vornehmen, zwischen denen starke inhaltliche Verbindungen bestehen (mit gegenseitigem oder wechselseitigem Charakter) brauchen nicht $\mathrm{zu}$ befürchten, dass sie gegen das Verbot von gemeinschaftlichen Testamenten verstoßen und die Verfügungen ungültig werden, es sei denn, dass diese in einer Urkunde enthalten worden sind 12 .

Sind die Verfügungen in einer Urkunde enthalten, so sind die Testamente ungültig und es ist ohne Bedeutung, ob zwischen den Verfügungen irgendwelche zusätzlichen inhaltlichen Verbindungen bestehen. Eine interessante Regelung gibt es im italienischen Recht, in dem das Kriterium der einheitlichen Urkunde mit zusätzlichen Elementen ergänzt wurde. Das italienische Verbot (Art. 589 ZGB) betrifft nämlich ausschließlich gemeinschaftliche gegenseitige Testamente (testamento reciproco) und gemeinschaftliche Testamente zugunsten eines Dritten (testamento congiuntivo), gilt aber nicht für gemeinschaftliche gleichzeitige Testamente (testamento simultaneo) ${ }^{13}$. Die Erblasser können also ihre Verfügungen in einer Urkunde verfassen, unter der Bedingung jedoch, dass diese nicht inhaltlich verbunden sind.

Art. 953, Rn. 10, S. 151 vertritt die Meinung, dass das auf dem polnischen Schiff oder im Flugzeug errichtete Testament den Charakter eines allographischen Testaments hat.

10 So treffend Przybyłowski, Fußnote 2, S. 21.

11 Nach der dominierenden Auffassung umfasst Art. 942 ZGB nicht das den Inhalt des mündlichen Testaments feststellende Schreiben, siehe z.B. J. Kremis, [in:] E. Gniewek, P. Machnikowski (Red.), Kodeks cywilny. Komentarz [Zivilgesetzbuch. Kommentar], Warszawa 2013, Art. 942, Rn. 4, S. 1598; Skowrońska-Bocian, Fußnote 9, S. 99 ff., These 5 und 6 zum Artikel 942; E. Niezbecka, [in:] A. Kidyba (Red.), Kodeks cywilny. Komentarz. Tom IV. Spadki [Zivilgesetzbuch. Kommentar. Vierter Band. Erbrecht], Warszawa 2012, S. 106 ff., These 3 zum Artikel 942.

12 So treffend Przybyłowski, Fußnote 2, S. 20.

13 Siehe dazu P. Kindler, Einführung in das italienische Recht, München 2008, S. 208; A. Zaccaria, [in:] S. Grundmann, A. Zaccaria (Red.), Einführung in das italienische Recht, Frankfurt a.M. 2007, S. 336-337. 
In der Rechtslehre wird auf zahlreiche Gründe für das Verbot von gemeinschaftlichen Testamenten im Erbrecht hingewiesen. Diese Gründe können grundsätzlich in drei Gruppen unterteilt werden. Erstens wird betont, dass das gemeinschaftliche Testament zu den Elementen im Widerspruch steht, die das Rechtsinstitut des Testaments von anderen Rechtsgeschäften unterscheiden. Bei einem gemeinschaftlichen Testament ist es nämlich nicht möglich, solche Grundsätze wie freie Widerrufbarkeit der Verfügungen ${ }^{14}$, Testierfreiheit ${ }^{15}$, Persönlichkeit ${ }^{16}$, Geheimhaltung ${ }^{17}$ oder Einseitigkeit der testamentarischen Willenserklärungen zu respektieren ${ }^{18}$.

14 Siehe dazu vor allem Przybyłowski, Fußnote 2, S. 11 ff.; siehe auch E. SkowrońskaBocian, Testament $w$ prawie polskim [Testament im polnischen Recht], Warszawa 2004, S. 207 ff.; K. Osajda, Testamenty wspólne [Gemeinschaftliche Testamente], Warszawa 2005, S. 89 ff.; W. Klyta, Testamenty wspólne [Gemeinschaftliche Testamente], Rejent [Notar] 2006, Nr. 2, S. 115.

15 So J. Gwiazdomorski, Wykładnia przepisów o testamencie na tle uchwaty składu siedmiu sędziów Sądu Najwyższego [Auslegung der Vorschriften über Testament aufgrund des Beschlusses der sieben Richter des Obersten Gerichts], Nowe Prawo [Neues Recht] 1973, Nr. 6, S. 830; S. Wójcik, [in:] J.St. Piątowski (Red.), System prawa cywilnego. Prawo spadkowe [System des Zivilrechts. Erbrecht], Wrocław-Warszawa-Kraków- Gdańsk-Łódź 1986, S. 190; S. Wójcik, F. Zoll, [in:] B. Kordasiewicz (Red.), System prawa prywatnego. Prawo spadkowe. Tom 10 [System des Privatrechts. Erbrecht. Zehnter Band], Warszawa 2009, S. 296; so aufgrund des venezuelischen Rechts siehe J. Basedow, K.J. Hopt, H.D. Kötz, [in:] U. Drobnig, M. Ferid, G. Kegel (Red.), Gutachten zum internationalen und ausländischen Privatrecht, Baden-Baden 1997, Nr. 39 a, S. 509; so über das italienische Verbot von gemeinschaftlichen Testamenten B. von Hoffmann, K. Thorn, Internationales Privatrecht einschließlich der Grundzüge des Internationalen Zivilverfahrensrechts, München 2007, S. 412; solch einen Standpunkt vertritt auch die portugiesische Rechtslehre, siehe E. Jayme, Zur Errichtung eines gemeinschaftlichen Testaments durch portugiesische Eheleute im Ausland, Praxis des Internationalen Privat- und Verfahrensrechts 1982, S. 210, Fußnote 5 und die dort genannte Literatur; siehe dazu Przybyłowski, Fußnote 2, S. 14; Skowrońska-Bocian, Fußnote 14, S. 208.

16 Siehe dazu Przybyłowski, Fußnote 2, S. 15, Fußnote 45 und die dort genannte Literatur; Wójcik, Fußnote 15, S. 190; Skowrońska-Bocian, Fußnote 14, S. 208; S. Wójcik, O niektórych uregulowaniach w prawie spadkowym. Uwagi de lege ferenda [Über manche Regelungen im Erbrecht. Anmerkungen de lege ferenda], [in:] L. Ogiegło, W. Popiołek, M. Szpunar (Red.), Rozprawy prawnicze. Ksiega pamiątkowa profesora Maksymiliana Pazdana [Juristische Abhandlungen. Gedächtnisschrift für Professor Maksymilian Pazdan], Kraków 2005, S. 1491.

17 Siehe dazu Przybyłowski, Fußnote 2, S. 16; Wójcik, Fußnote 15, S. 190; Wójcik, Fußnote 16, S. 1491; siehe jedoch Osajda, Fußnote 14, S. 94, der feststellt, dass: „Nach polnischem Recht steht eine solche Eigenschaft dem Akt des letzten Willens nicht zu“.

18 So Przybyłowski, Fußnote 2, S. 13, in Verbindung mit der auf S. 16 dargestellten Stellungnahme; Wójcik, Fußnote 15, S. 190; Wójcik, Fußnote 16, S. 1491; Wójcik, Zoll, Fußnote 15, S. 296; so über das italienische Verbot von gemeinschaftlichen Testamenten P. Hay, Internationales Privat- und Zivilverfahrensrecht, München 2007, S. 249 und das Bayerische Oberste Landesgericht in Zivilsachen in der Entscheidung vom 13.12.1957, Entscheidungen des Bayerischen Obersten Landesgerichts in Zivilsachen 1957, S. 384 und die dort genannte Literatur. 
Zweitens werden Befürchtungen geäußert, dass eine positive Regelung des analysierten Rechtsinstituts sich ungünstig auf zwischenmenschliche Beziehungen (im weiten Sinne) auswirken wird. Sie führt nämlich dazu, dass das Risiko von gegenseitiger Erschleichung der Verfügungen durch gemeinschaftlich testierende Erblasser steigen ${ }^{19}$ und die Beziehung zwischen ihnen durch die für Vertragsverhältnisse charakteristische Regel der Gegenseitigkeit ${ }^{20}$ dominiert wird; auch das Risiko von verwickelten Gerichtsstreitigkeiten zwischen den Erblassern wird steigen ${ }^{21}$.

Drittens wird das gemeinschaftliche Testament für ein überflüssiges Institut gehalten ${ }^{22}$, welches die steigende Zahl der ungültigen Testamente zur Folge hat ${ }^{23}$.

Die oben angeführten Argumente werden im Allgemeinen von der polnischen Rechtslehre nicht angezweifelt ${ }^{24}$.

\section{VERBOT VON GEMEINSCHAFTLICHEN TESTAMENTEN IM INTERNATIONALEN PRIVATRECHT}

Die Frage nach dem Rechtscharakters vom Verbot gemeinschaftlicher Testamente ist aus zwei grundlegenden Gründen von Bedeutung. Erstens kann das polnische Gericht, wenn es den Rechtscharakter der Norm aus Art. 942 ZGB kennt, das fremde Verbot von gemeinschaftlichen

19 Siehe dazu Przybyłowski, Fußnote 2, S. 15; Fußnote 45 und die dort genannte Literatur.

20 Siehe zum italienischen Verbot von gemeinschaftlichen Testamenten S. Grundmann, Zur Errichtung eines gemeinschaftlichen Testaments durch italienische Ehegatten in Deutschland, Praxis des Internationalen Privat- und Verfahrensrechts 1986, S. 96 und die dort genannte Literatur, sowie die Entscheidung des Bayerischen Obersten Landesgerichts in Zivilsachen vom 13.12.1957, Entscheidungen des Bayerischen Obersten Landesgerichts in Zivilsachen 1957, S. 384 und die dort genannte Literatur.

21 Siehe dazu Przybyłowski, Fußnote 2, S. 15 und die dort genannte Literatur; so Wójcik, Fußnote 15, S. 190; Wójcik, Fußnote 16, S. 1491.

22 Siehe dazu Przybyłowski, Fußnote 2, S. 16.

23 Siehe dazu Ibidem, S. 16, Fußnote 51, der die Begründung des tschechoslowakischen Entwurfs des Zivilgesetzbuches veröffentlicht in: Das Bürgerliche Gesetzbuch für die Čechoslovakische Republik, Reichenberg 1924, S. 514 anführt; Osajda, Fußnote 14, S. 94.

24 Eine Ausnahme bildet die Meinung von Osajda, Fußnote 14, S. 91 ff., der alle Argumente, die in der Rechtslehre zur Begründung der Regelung von Verbot gemeinschaftlicher Testamente angeführt werden, in Zweifel zieht. 
Testamenten ${ }^{25}$ in einer Sache mit internationalem Element nach legis fori qualifizieren ${ }^{26}$. Zweitens ist die durch die polnische Rechtslehre und Rechtsprechung getroffene Beurteilung des Rechtscharakters vom polnischen Verbot gemeinschaftlicher Testamente von Bedeutung für Gerichte anderer Länder (z.B. deutsche Gerichte), die ad casum das Rechtsnatur vom polnischen Verbot gemeinschaftlicher Testamente bestimmen müssen ${ }^{27}$.

In beiden Fällen müssen die Gerichte die sog. Qualifikation vom Verbot gemeinschaftlicher Testamente vornehmen ${ }^{28}$, es also einem der zwei Statute zuordnen, die zur Beurteilung der Gültigkeit des Testaments angewandt werden, d.h. dem Formstatut oder dem hypothetischen Erbstatut ${ }^{29}$. Ist das Verbot lediglich eine weitere Vorschrift zur Regelung der Form von einem Testament, dann gehört es zum Formstatut. Bei der Annahme, dass das Verbot eine materiellrechtliche Norm zur Regelung des zulässigen Inhalts von einem Testament ist, ist das Verbot dem hypothetischen Erbstatut zuzuordnen.

25 Das Verbot von gemeinschaftlichen Testamenten regeln: Art. 968 Französisches ZGB; Art. 968 Belgisches ZGB; Art. 589 Italienisches ZGB; Art. 4.3.5.1 Niederländisches ZGB; Art. 2181 Portugiesisches ZGB; Art. 857 Rumänisches ZGB; Art. 669 Spanisches ZGB; Art. 975 Japanisches ZGB; Art. 3618 Argentinisches ZGB; Art. 1630 Brasilianisches ZGB; Art. 1003 Chilienisches ZGB; Art. 1063 Equadorisches ZGB; Art. 1059 Kolumbianisches ZGB; Art. 1295 Mexikanisches ZGB; Art. 1612 Paraguayisches ZGB; Art. 2123 Portorikanisches ZGB; Art. 781 Uruguayisches ZGB; Art. 835 Venezuelisches ZGB; Art. 818 Philippinisches ZGB - siehe R. Süß, Das Verbot gemeinschaftlicher Testamente im Internationalen Erbrecht, Praxis des Internationalen Privat- und Verfahrensrechts 2002, Heft 1, S. 23, Fußnote 15.

26 Für die Qualifikation von fremden Verboten gemeinschaftlicher Testamente nach legis fori spricht sich A. Mączyński aus, Dziedziczenie testamentowe w prawie prywatnym międzynarodowym. Ustawowe $i$ konwencyjne unormowanie problematyki formy [Testamentarische Erbfolge. Gesetzliche und konventionelle Regelung der Formproblematik], Warszawa-Kraków 1967, S. 104.

27 Siehe dazu M. Margoński, Ważność testamentów sporządzonych przez obywateli polskich $w$ sprawach spadkowych z elementem transgranicznym [Gültigkeit der von polnischen Bürgern errichteten Testamente in Erbsachen mit grenzüberschreitendem Element], Przegląd Sądowy [Gerichtsumschau] 2010, Nr. 10, S. 66.

28 Die Qualifikation gehört zu den besonders strittigen Fragen - siehe L. Raape, Internationales Privatrecht, Berlin und Frankfurt a.M. 1961, S. 426, nach dessen Meinung "das gemeinsame Testament gehört zu den Zankäpfeln des IPR“; siehe auch Mączyński, Fußnote 26, S. 100.

29 Eine solche Definition der Qualifikation nimmt C.F. Nordmeier an, Zulässigkeit und Bindungswirkung gemeinschaftlicher Testamente im Internationalen Privatrecht. Unter besonderer Berücksichtigung der Rechte portugiesischsprachiger Länder, Tübingen 2008, S. 64. 
Das polnische Gericht wird durch Qualifikation des fremden Verbots gemeinschaftlicher Testamente nach legis fori, also nach dem am Sitz des erkennenden Gerichts geltenden Recht, d.h. nach dem polnischen Recht, es dem Formstatut zuordnen, wenn es anerkennt, dass im polnischen Recht das Verbot gemeinschaftlicher Testamente zu der Gruppe der Vorschriften gehört, die die Form von einem Testament regeln. Wenn es dagegen anerkennt, dass das polnische Verbot gemeinschaftlicher Testamente sich auf den Inhalt des Testaments bezieht, wird die fremde Verbotsnorm dem hypothetischen Erbstatut zugeordnet.

Bei Berücksichtigung des Inhalts des Haager Übereinkommens vom 5. Oktober 1961 zur Rechtskollision bei der Form der testamentarischen Verfügungen ${ }^{30}$, wie auch der Verordnung des Europäischen Parlaments und des Rates vom 4. Juli 201231, die dem Erblasser große Freiheit bei der Wahl der Testamentsform lassen, kann sich erweisen, dass das dem Formstatut zugeordnete Verbot gemeinschaftlicher Testamente ad casum bei der Beurteilung der Gültigkeit des Testaments bezüglich der Form überhaupt keine Anwendung findet. Laut Art. 1 des Übereinkommens ist die testamentarische Verfügung gültig bezüglich der Form, wenn sie vereinbar ist mit dem Recht des Ortes, an dem der Erblasser die Verfügung getätigt hat, oder mit dem Recht, das in dem Staat gilt, dessen Staatsbürger der Erblasser entweder zum Zeitpunkt der Verfügungstätigung oder zum Zeitpunkt des Todes ist, oder des Ortes, an dem der Erblasser seinen Wohnsitz zum Zeitpunkt der Verfügungstätigung oder zum Zeitpunkt des Todes hatte, oder des Ortes des Aufenthalts zum Zeitpunkt der Verfügungstätigung oder zum Zeitpunkt des Todes, in Bezug auf Grundstücke - ihre Lage. In der Folge, wenn das Verbot zu den Formvorschriften gezählt wird, kann die Beurteilung der Gültigkeit von einem gemeinschaftlichen Testament positiv sein, wenn nur den Erblassern laut Haager Übereinkommen das Recht zustand, die für das gemeinschaftliche Testament angemessene Form $\mathrm{zu}$ nutzen. Zum

\footnotetext{
30 Dz.U. [Gesetzblatt] 1969, Nr. 34, Pos. 284.

31 Verordnung (EU) Nr. 650/2012 des Europäischen Parlaments und des Rates vom 4.07.2012 über die Zuständigkeit, das anzuwendende Recht, die Anerkennung und Vollstreckung von Entscheidungen und die Annahme und Vollstreckung öffentlicher Urkunden in Erbsachen sowie zur Einführung eines Europäischen Nachlasszeugnisses, Dz.U. [Gesetzblatt] UE L 201/107.
} 
Beispiel ist als in formeller Hinsicht gültig (gemäß Art. 1 Pkt. 1 des Übereinkommens) das von Eheleuten in Deutschland errichtete gemeinschaftliche Testament anzuerkennen. An dem Ort, an dem die gemeinschaftlichen Verfügungen vorgenommen wurden, gilt nämlich eine positive Regelung des Rechtsinstituts des gemeinschaftlichen Testaments, was unter formellem Gesichtspunkt den Erblassern erlaubt, ihre Verfügungen in einer Urkunde zu enthalten. Es soll erneut betont werden, dass es sich natürlich nur um solche Situationen handelt, wenn das jeweilige Verbot gemeinschaftlicher Testamente dem Formstatut zugeordnet wurde. Die Zuordnung des Verbots zum hypothetischen Erbstatut hat immer die Ungültigkeit der Verfügungen zur Folge, es sei denn, dass in dem erwähnten Statut eine andere Sanktion für die Verletzung der Verbotsnorm vorgesehen ist.

Es entsteht also die Frage, wie das polnische Verbot von gemeinschaftlichen Testamenten $\mathrm{zu}$ qualifizieren ist. Es soll erwogen werden, $\mathrm{ob}$ es eine Norm ist, die $\mathrm{zu}$ den Vorschriften über die Testamentsform gezählt werden soll, ob aber Art. 942 ZGB eine Regelung des zulässigen Testamentsinhalts ist. Die Frage scheint nicht einfach $\mathrm{zu}$ beantworten $\mathrm{zu}$ sein, und die Rechtsprechung ist diesbezüglich geteilt. Nach der vorherrschenden Auffassung ist das Verbot gemeinschaftlicher Testamente eine Vorschrift mit rein formellem Charakter, die die in Art. 949-958 enthaltene Regelung der zulässigen Testamentsformen ergänzt ${ }^{32}$. In erster Linie wird auf die Art und Weise der Auslegung von Art. 942 ZGB hingewiesen, der laut der dominierenden Auffassung ausschließlich schriftlich verfasste Testamente umfasst, und die

32 Mączyński, Fußnote 26, S. 103; Margoński, Fußnote 27, S. 67; K. Osajda, [in:] K. Osajda (Red.), Kodeks cywilny. Komentarz. Tom III. Spadki [Zivilgesetzbuch. Kommentar. Band III. Erbrecht], Warszawa 2013, Art. 942, Rn. 34, S. 273; Niezbecka, Fußnote 11, S. 107, These 6 zum Artikel 942; die Zulässigkeit der gemeinschaftlien Testamente ist die Frage des Testamentsform - so unzutreffend M. Pazdan, Czynności notarialne w międzynarodowym prawie spadkowym [Notarielle Handlungen im internationalen Erbrecht], Rejent [Notar] 1998, Nr. 4, S. 107; J. Pazdan, [in:] M. Pazdan (Red.), System prawa prywatnego. Prawo prywatne międzynarodowe. Tom 20A [System des Privatrechts. Internationales Privatrecht. Band 20A], Warszawa 2014, S. 776; J. Gołaczyński, Prawo prywatne międzynarodowe [Internationales Privatrecht], Warszawa 2011, Rn. 513, S. 322; siehe dazu M. Margoński, [in:] K. Osajda (Red.), Konwencja haska dotycząca kolizji praw w przedmiocie formy rozrządzeń testamentowych. Komentarz [Hagger Übereinkommen über das auf die Form letztwilliger Verfügungen anzuwendende Recht. Kommentar], Warszawa 2015, Art. 4. 
mündlichen Testamente und Schreiben zur Festlegung ihres Inhalts nicht betrifft ${ }^{33}$. Wie es scheint, gibt es keine ausreichende Begründung dafür, aus der Tatsache der beschränkten Anwendung des Verbots ein Argument für dessen formellen Charakter $\mathrm{zu}$ machen. Erblasser können ihre Verfügungen auf verschiedene Weisen verbinden, aber nicht jede stellt eine Gefährdung der Interessen der Erblasser dar. Die Analyse von Art. 942 ZGB führt zu der Schlussfolgerung, dass der Gesetzgeber eine solche Gefährdung ausschließlich in schriftlich verfassten Testamenten erblickte. Aber nur aus dem Grund, dass der Gesetzgeber in mündlichen Testamenten solche Gefährdungen nicht gesehen hat, kann dem Verbot der materiellrechtliche Charakter nicht entzogen werden. Es fällt auch schwer, in dieser Beschränkung der Anwendung von Art. 942 ZGB irgendeine Widersprüchlichkeit der rechtsetzenden Tätigkeit des Gesetzgebers zu sehen, der doch alle Schriftformen einheitlich behandelt. Eine andere Einstellung zum mündlichen Testament ergibt sich dagegen aus der Besonderheit dieser Testamentsform, deren Eigenschaften die Verbindung der Verfügungen auf eine die Erblasser gefährdende Weise, also in einer Urkunde, nicht zulassen.

Auf den formalrechtlichen Charakter des Verbots soll auch die durch Rechtsprechung zugelassene Möglichkeit der Konversion des ungültigen gemeinschaftlichen allographischen Testaments in zwei mündliche Testamente hinweisen ${ }^{34}$. Daraus sollen aber keine zu weit gehenden Schlussfolgerungen gezogen werden. Auf diese Art und Weise werden Verfügungen nämlich nur in ausgesprochenen Ausnahmesituationen aufrechterhalten. Darüber hinaus wird die Konversion für alle ungültigen Rechtsgeschäfte angewandt, unabhängig von Gründen ihrer Ungültigkeit. Es handelt sich also eher um die rechtliche Natur der Konversion, bei der die Möglichkeit ihrer Anwendung nicht von ratio legis der Vorschrift, die der Grund für die Ungültigkeit einer Handlung ist, abhängig gemacht wird.

Darüber hinaus soll auf den formalrechtlichen Charakter des Verbots von gemeinschaftlichen Testamenten die Tatsache hinweisen, dass das Verbot sich lediglich auf die in einer Urkunde enthaltenen Verfügungen

\footnotetext{
33 Margoński, Fußnote 27, S. 67.

34 Ibidem, S. 67.
} 
bezieht, nicht aber auf den Inhalt der Verfügungen selbst ${ }^{35}$. Es ist erneut zu betonen, dass eine solche Regelung Reaktion auf gewisse Gefährdungen ist, denen der Gesetzgeber wirksam vorbeugt. Die Bedeutung von diesen Gefährdungen soll nicht nur deshalb verringert werden, dass der Gesetzgeber, um den Umfang des Verbots zu bestimmen, das Kriterium der Urkunde und nicht des Rechtsgeschäfts verwendete. Die Verwendung des Begriffs Testament im Sinne von Urkunde, kann nicht a priori aus Art. 942 ZGB eine Norm mit für Formvorschriften typischen Eigenschaften machen.

Es wird betont, dass die Antwort auf die Frage nach dem Rechtscharakter des Verbots sich nur schwer in Ergebnissen der systematischen Auslegung finden lässt, da diese nicht eindeutig sind. Es fällt aber schwer, einer solchen Ansicht zuzustimmen, wenn Art. 942 ZGB zu den allgemeinen Vorschriften zum Testament und nicht zu den Vorschriften über die Testamentsform gezählt wurde. Alle allgemeinen Vorschriften zum Testament, was hervorgehoben werden soll, sind dem hypothetischen Erbstatut zuzuordnen. Dies bedeutet, dass sie einen materiellrechtlichen Charakter haben. Die Regelung des Verbots unter den zu diesem Status gehörenden Vorschriften soll für die Beurteilung des Rechtscharakters des Verbots ausschlaggebend sein. Die Anerkennung, dass das Verbot einen formalen Charakter hat, wäre nur dann möglich, wenn Art. 942 ZGB den Vorschriften über die Form des Testaments, also den Vorschriften des Formstatuts, zugeordnet wäre. So ist es aber nicht und das muss in Betracht gezogen werden.

In der deutschen Rechtsliteratur werden viele Beispiele genannt, in denen die ausländische Rechtslehre und Rechtsprechung die Qualifikation der eigenen Verbote von gemeinschaftlichen Testamenten im Widerspruch zum eindeutigen Ergebnis der systematischen Auslegung vornehmen ${ }^{36}$. Es soll vermeintlich ein Argument dafür sein, dass die durch ein deutsches Gericht durchgeführte systematische Auslegung des fremden Verbots von gemeinschaftlichen Testamenten $\mathrm{zu}$ seiner falschen Qualifikation führen kann, wobei als falsch eine Qualifikation anerkannt wird, die im

35 Niezbecka, Fußnote 11, S. 107, These 6 zum Art. 942.

36 Süß, Fußnote 25, S. 24. 
Widerspruch zu der Stellung der ausländischen Rechtsprechung steht ${ }^{37}$. Hier soll aber die Frage gestellt werden, welche Bedeutung hat dieser Umstand für die Beurteilung, ob das Ergebnis der systematischen Auslegung durch die polnische Rechtsprechung im Fall des polnischen Verbots von gemeinschaftlichen Testamenten berücksichtigt werden sollis8. Das angeführte rechtsvergleichende Argument bezieht sich auf ein anderes Problem, d.h. Qualifikation fremder Verbote von gemeinschaftlichen Testamenten durch deutsche Gerichte und ist sicherlich irrelevant für die Beurteilung des Rechtscharakters des polnischen Verbots von gemeinschaftlichen Testamenten. Es ist verständlich, dass die deutsche Rechtslehre bei der Qualifikation fremder Verbote von gemeinschaftlichen Testamenten sich von den Ergebnissen der systematischen Auslegung distanziert, da sie keine Antwort auf die Frage gibt, wie das Verbot durch die Rechtsprechung des jeweiligen Staates qualifiziert wird. So sieht der Prozess der Qualifikation des Verbots im deutschen Recht aus, in dem, in Anlehnung an das französische Recht, das Verbot von gemeinschaftlichen Testamenten nicht expresis verbis geregelt worden und die Qualifikation nach legis fori im klassischen Sinne einfach nicht möglich ist. Die Antwort auf die Frage nach dem Statut des Verbots wird also im Recht des Staates gesucht, aus dem das Verbot stammt ${ }^{39}$. Darum aus der Sicht des deutschen Gerichts, das das fremde Verbot qualifiziert und darauf abzielt, dass das Ergebnis der Qualifikation der in dem jeweiligen Staat geltenden Qualifikation des Verbots entspricht, ist die systematische Auslegung ausgesprochen ungeeignet. Das deutsche Gericht weiß nämlich nicht,

\footnotetext{
37 Siehe Margoński, Fußnote 27, S. 66.

38 Eine positive Antwort auf diese Frage scheint M. Margoński zu geben; Ibidem, S. 66.

39 S. Grundmann, Qualifikation gegen die Sachnorm, München 1985, S. 125; Süß, Fußnote 25, S. 26; Jayme, Fußnote 15, S. 210 vertreten den Standpunkt, dass Qualifikation des fremden Verbots im deutschen Recht in Anlehnung auf legis causae erfolgt. H. Dörner, [in:] J. Kropholler (Red.), J. von Staudingers Kommentar zum Bürgerlichen Gesetzbuch mit Einführungsgesetz und Nebengesetzen. Einführungsgesetz zum Bürgerlichen Gesetzbuch/IPR Art. 25, 26 EGBGB (Internationales Erbrecht), Berlin 2007, Art. 25, Rn. 326, S. 221 und G. Kegel, Zur Bindungswirkung an das gemeinschaftliche Testament im deutschen internationalen Privatrecht, [in:] K. Carstens, H. Peters (Red.), Festschrift Herman Jahrreiss zu seinem siebzigsten Geburtstag 19. August 1964 - gewidmet, Köln-Berlin-Bonn-München 1964, S. 154; F. Firsching, Die 9. Haager Konferenz, Rabels Zeitschrift für ausländisches und internationales Privatrecht 1962, Nr. 63, S. 423 behaupten dagegen, dass im deutschen Recht die Qualifikation trotzdem nach legis fori erfolgt.
} 
ob in dem jeweiligen Staat bei der Beurteilung des Rechtscharakters des Verbots seine systematische Auslegung überhaupt berücksichtigt worden ist.

Die Frage der Nichtanwendung der systematischen Auslegung in anderen Staaten, in denen das Verbot gemeinschaftlicher Testamente gilt, soll jedoch im rechtsvergleichenden Kontext berücksichtigt werden. Beim Versuch, die Frage zu beantworten, ob die systematische Auslegung zur Beurteilung des Rechtscharakters vom Verbot gemeinschaftlicher Testamente in anderen Ländern angewandt wird, soll festgestellt werden, dass ihr Ergebnis oft nicht berücksichtigt wird. Da entsteht aber der Zweifel, ob das ein Beweis für die Ungeeignetheit der systematischen Auslegung für die Beurteilung des Rechtscharakters vom polnischen Verbot gemeinschaftlicher Testamente oder lediglich eine Bestätigung von bewusster und gegen die geltenden Regeln verstoßenden Unterschätzung der Ergebnisse dieser Auslegung ist, um ein früher angenommenes Ziel zu erreichen. Und dieses Ziel ist es, die gemeinschaftlichen Verfügungen sogar auf Kosten der contra legem Entscheidung zu retten. Die Achtung für den Gesetzgeber erfordert, dass die Ergebnisse der systematischen Auslegung berücksichtigt und als bindend betrachtet werden ${ }^{40}$. Die Zuordnung des polnischen Verbots von gemeinschaftlichen Testamenten ist der einzige Umstand von normativem Charakter, der eindeutig auf den Rechtscharakter hinweist. Ergebnisse der rechtsvergleichenden Analyse können nicht dazu führen, dass dieser Umstand ignoriert wird.

Um den formalen Charakter von Art. 942 ZGB zu begründen, werden auch die Gründe für die Einführung des Verbots von gemeinschaftlichen Testamenten in die polnische Rechtsordnung absichtlich übergangen. Es wird nämlich festgestellt, dass sie in diesem Fall irrelevant sind ${ }^{41}$, da derzeit lediglich die Argumente wiederholt werden, die gegen das Institut des gemeinschaftlichen Testaments bei den Arbeiten an den Kodifizierungen im 19. Jh. angeführt worden sind ${ }^{42}$. Für diesen Standpunkt scheint es aber keine ausreichende Argumentation $\mathrm{zu}$ geben. Es ist vollkommen natürlich, sogar notwendig, auf das umfangreiche

40 Das Ergebnis der systematischen Auslegung als bindend erklärt auch J. Balicki, Problemy kolizyjne prawa spadkowego [Kollisionsprobleme im Erbrecht], Warszawa 1963, S. 90.

41 So Mączyński, Fußnote 26, S. 104.

42 Margoński, Fußnote 27, S. 66. 
theoretische Wissen zum Institut des gemeinschaftlichen Testaments zurückzugreifen. Der Meinung, dass die angeführten Argumente sich „überlebt haben“, ist nur schwer Recht zu geben. Sie müssen selbstverständlich einer kritischen Analyse unterzogen werden, um beurteilen zu können, ob die Regelung des Verbots gemeinschaftlicher Testamente begründet war. Erst wenn man alle Umstände berücksichtigt, kann das Verbot entweder den materiellrechtlichen oder den formalrechtlichen Normen zugeordnet werden.

Nach der dominierenden Auffassung der deutschen Rechtslehre ist das Verbot den Vorschriften über die Testamentsform zuzuschreiben, wenn seine Funktionen darin bestehen, das Errichten von Testamenten in derselben Urkunde $\mathrm{zu}$ verhindern 43 , den Willen des Erblassers unzweifelhaft festzustellen, die Authentizität der Willenserklärung und ihre Beweisbarkeit sicherzustellen ${ }^{44}$, undurchdachte und übereilte Testamentserrichtung zu verhindern (Warnfunktion)45, zur Einhaltung der Sorgfalt bei Testamentserrichtung zu veranlassen ${ }^{46}$ oder die Einhaltung des Grundsatzes unitas actus sicherzustellen ${ }^{47}$. Dieser Grundsatz besteht darin, den Rechtsakt ununterbrochen in derselben Zeit und an demselben Ort vorzunehmen 48 , wobei bei einem gemeinschaftlichen Testament das Testieren ohne seine Verletzung nicht möglich ist. Die

\footnotetext{
43 A. Staudinger, [in:] R. Schulze (Red.), Bürgerliches Gesetzbuch. Handkommentar, BadenBaden 2007, Art. 26 EGBGB, Rn. 9, S. 2316; von Hoffmann, Thorn, Fußnote 15, S. 412; vgl. Nordmeier, Fußnote 29, S. 66.

$44 \quad$ K. Schurig, [in:] G. Kegel (Red.), Soergel Bürgerliches Gesetzbuch mit Einführungsgesetz und Nebengesetzen. Band 10. Einführungsgesetz, Stuttgart 1996, Art. 26, Rn. 23, S. 1372; H. Dörner, Fußnote 38, Art. 25, Rn. 325, S. 220; G. Kegel, K. Schurig, Internationales Privatrecht, München 2004, S. 1013; J. Kropholler, Internationales Privatrecht, Tübingen 2006, S. 447; H. Schack, Gemeinschaftliche Testamente im Internationalen Privatrecht, [in:] H. Schack (Red.), Gedächtnisschrift für Alexander Lüderitz, München 2000, S. 664; Süß, Fußnote 25, S. 23; R. Freitag, [in:] H. Prütting, G. Wegen, G. Weinreich (Red.), BGB Kommentar, Köln 2009, Art. 25 EGBGB, Rn. 20, S. 3136; L. Kroiß, [in:] B. Dauner-Lieb, T. Heidel, G. Ring (Red.), Anwaltskommentar BGB, Bonn 2005, Art. 25, Rn. 20, S. 2063; vgl. Nordmeier, Fußnote 29, S. 66.

$45 \quad$ Kroiß, Fußnote 43, Art. 25, Rn. 20, S. 2063; vgl. Nordmeier, Fußnote 29, S. 66.

46 K. Firsching, [in:] D. Henrich (Red.), J. von Staudingers Kommentar zum Bürgerlichen Gesetzbuch mit Einführungsgesetz und Nebengesetzen, Einführungsgesetz zum Bürgerlichen Gesetzbuch Art. 24-28 aF; 5, 6 nF, Berlin 1991, Vor § 24-26, Rn. 271, S. 120; Kegel, Fußnote 38, S. 155; Nordmeier, Fußnote 29, S. 66.

47 Firsching, Fußnote 45, S. 120; Kegel, Fußnote 38, S. 155; vgl. Nordmeier, Fußnote 29, S. 66 .

48 W. Wołodkiewicz, [in:] W. Wołodkiewicz (Red.), Prawo rzymskie. Stownik encyklopedyczny [Römisches Recht. Enzyklopädisches Lexikon], Warszawa 1986, S. 152.
} 
Testamentserrichtung durch einen Erblasser ist nämlich ein durch die Rechtshandlungen von Todes wegen (Testament) des anderen Erblassers "unterbrochener" Prozess.

Das Verbot soll hingegen den Vorschriften über Testamentsinhalt zugeordnet werden, wenn es die gegenseitigen Rechtsfolgen verhindert ${ }^{49}$, in Form von Wechselbezüglichkeit und Bindungswirkung50, die Widerruflichkeit des Testaments nach dem Tode von einem der Erblasser sicherstellt ${ }^{51}$, die Einseitigkeit des Testaments garantiert ${ }^{52}$, die Testierfreiheit gewährleistet ${ }^{53}$ oder die Entstehung des Bindungsgefühls verhindert, worunter die Unmöglichkeit der Änderung der gemeinschaftlich vorgenommenen Verfügungen verstanden wird 54 .

Die Funktion des Verbots, die darin besteht, bei der Testamentserrichtung die Beeinflussung des Erblassers zu verhindern ist umstritten ${ }^{55}$. Manche glauben, dass diese Funktion typisch für Formvorschriften ist, andere dagegen meinen, dass ihre Existenz für ihre Zuordnung zu den Vorschriften über den Testamentsinhalt ausschlaggebend ist ${ }^{56}$.

Das im deutschen Recht dargestellte Konzept der Unterteilung der Gründe für das Verbot gemeinschaftlicher Testamenten ist überzeugend

\footnotetext{
49 Staudinger, Fußnote 42, Art. 26 EGBGB, Rn. 9, S. 2316; Nordmeier, Fußnote 29, S. 66. 50 Freitag, Fußnote 43, Art. 25 EGBGB, Rn. 20, S. 3136; Kroiß, Fußnote 43, Art. 26 EGBGB, Rn. 32, S. 2063; von Hoffmann, Thorn, Fußnote 15, S. 411; Lange, Kuchinke, Erbrecht, München 2001, S. 421; Nordmeier, Fußnote 29, S. 66.

51 Kropholler, Fußnote 43, S. 447; Schack, Fußnote 43, S. 664; Süß, Fußnote 25, S. 23; Nordmeier, Fußnote 29, S. 66.

52 Entscheidung des Oberlandesgerichts in Frankfurt a.M. vom 17.05.1985, Praxis des Internationalen Privat- und Verfahrensrechts 1986, S. 111 ff.; Kroiß, Fußnote 43, Art. 26 EGBGB, Rn. 32, S. 2063; Hay, Fußnote 18, S. 249; Nordmeier, Fußnote 29, S. 66.

53 Schurig, Fußnote 43, Art. 26, Rn. 23, S. 1372; Dörner, Fußnote 38, Art. 25, Rn. 325, S. 220; S. Lorenz, [in:] H.G. Bamberger, H. Roth (Red.), Kommentar zum Bürgerlichen Gesetzbuch. Band 3, München 2012, Art. 26 EGBGB, Rn. 5, S. 2985; T. Wachter, [in:] H. Flick, D.J. Piltz (Red.), Der Internationale Erbfall, München 2008, S. 27; Süß, Fußnote 25, S. 23; vgl. Nordmeier, Fußnote 29, S. 66.

54 Entscheidung des Oberlandesgerichts in Frankfurt a.M. vom 17.05.1985, Praxis des Internationales Privat- und Verfahrensrechts 1986, Heft 2, S. 112; von Hoffmann, Thorn, Fußnote 15, S. 412; Hay, Fußnote 18, S. 249; Nordmeier, Fußnote 29, S. 67.

55 Süß, Fußnote 25, S. 22.

56 Ein Anhänger des ersten Konzepts ist Kropholler, Fußnote 43, S. 447; das andere Konzept befürworten: Schack, Fußnote 43, S. 664; Kegel, Schurig, Fußnote 43, S. 1013; Kroiß, Fußnote 43, Art. 26 EGBGB, Rn. 32, S. 2063; Dörner, Fußnote 38, Art. 25, Rn. 325, S. 220; von Hoffmann, Thorn, Fußnote 15, S. 412; Schurig, Fußnote 43, Art. 26, Rn. 23, S. 1327; Nordmeier, Fußnote 29, S. 67.
} 
und kann erfolgreich in das polnische Recht übertragen werden, um eine funktionale Auslegung des polnischen Verbots gemeinschaftlicher Testamente durchzuführen. Selbst eine flüchtige Analyse der schon genannten Gründe der Regelung des polnischen Verbots gemeinschaftlicher Testamente muss $\mathrm{zu}$ der Schlussfolgerung führen, dass es einen materiellrechtlichen Charakter hat ${ }^{57}$.

\section{SCHLUSSBEMERKUNGEN}

Im Licht der angeführten Argumente scheint das Konzept des Verbots als eines rein formellen Instituts wenig überzeugend $\mathrm{zu}$ sein. Die Liberalisierung der Auffassung findet keine Begründung in dem im Erbrecht geltenden Grundsatz favor testamenti. Seine Anwendung kann nämlich nicht zu einer solchen Interpretation des Verbots führen, die eine grobe Verletzung des Gesetzes darstellt.

Es fällt schwer einzuschätzen, welche Bedeutung für die Beurteilung des Rechtscharakters des polnischen Verbots von gemeinschaftlichen Testamenten die Verordnung des Europäischen Parlaments und des Rates vom 4. Juli $2012^{58}$ hat, in der der Begriff des gemeinschaftlichen Testaments, wie auch des gegenseitigen Testaments (siehe Art. 3 Abs. 1 lit. b und c) verwendet worden ist. Diese Unterteilung soll den konkludenten Willen des EU-Gesetzgebers zum Ausdruck bringen, dass die in einer Urkunde enthaltenen gegenseitigen Testamente immer auf die Vereinbarkeit mit dem hypothetischen Erbstatut, die Verfügungen dagegen, die nicht gegenseitig sind, auf die Vereinbarkeit mit dem Formstatut geprüft werden sollen ${ }^{59}$. Über die Richtigkeit einer solche

\footnotetext{
57 Auf materielles Charakter des Verbots macht auch M. Sośniak aufmerksam, Z kolizyjnej problematyki spadkobrania testamentowego [Aus Kollisionsproblematik der testamentarischen Erbfolge], Studia Prawnicze [Juristisches Studium] 1976, Nr. 1-2, S. 9.

58 Verordnung (EU) Nr. 650/2012 des Europäischen Parlaments und des Rates vom 4.07.2012 über die Zuständigkeit, das anzuwendende Recht, die Anerkennung und Vollstreckung von Entscheidungen und die Annahme und Vollstreckung öffentlicher Urkunden in Erbsachen sowie zur Einführung eines Europäischen Nachlasszeugnisses, Dz.U. [Gesetzblatt] UE L 201/107.

59 Siehe dazu A. Wysocka-Bar, Prawo właściwe dla formy rozporządzeń na wypadek śmierci wedtug rozporzadzenia spadkowego [Anwendbares Recht für die Form der Verfügungen von Todes wegen gemäß Erbschaftsverordnung], [in:] M. Pazdan, J. Górecki (Red.), Nowe europejskie prawo spadkowe [Neues europäisches Erbrecht], Warszawa 2015, S. $141 \mathrm{ff}$.
} 
Hypothese lässt sich schwer diskutieren, da in der Verordnung von einem von normativen Bedingungen vieler Länder getrennten „Vereinbarung aufgrund gegenseitiger Testamente“ (siehe Art. 3 Abs. 1 lit. c) die Rede ist. Diese Frage wird sicherlich noch diskutiert werden, aber jetzt ist es noch zu früh, das Endergebnis dieser Diskussion vorauszusehen. Vorerst soll man für die einheitliche, d.h. materielle Auffassung des Rechtscharakters des polnischen Verbots von gemeinschaftlichen Testamenten plädieren. 
